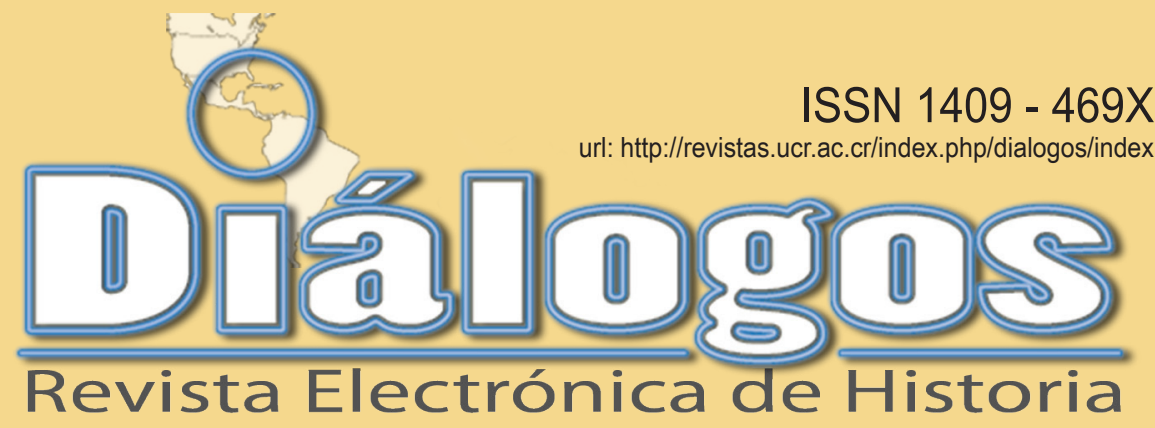

Escuela de Historia. Universidad de Costa Rica Volumen 15 Especial Región Occidente de Costa Rica - Octubre, 2014

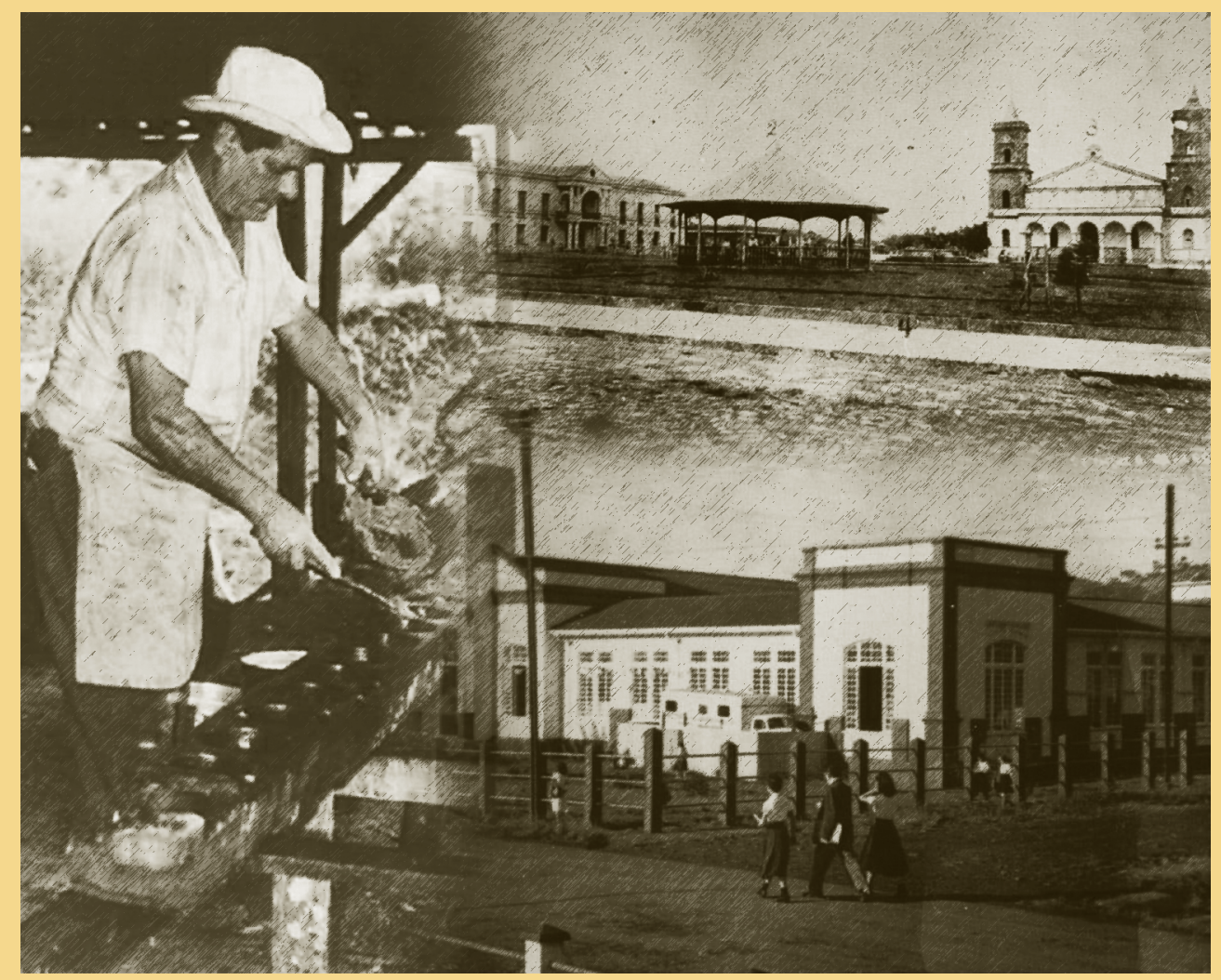

Director de la Revista: Dr. Juan José Marín Hernández juan.marinhernandez@ucr.ac.cr

Editor académico: Dr. David Díaz Arias - david.diaz@ucr.ac.cr

Editores invitados: M.Sc. William Solórzano Vargas - william.solorzano@ucr.ac.cr M.Ed. Maynor Badilla Vargas - mainor.badilla@ucr.ac.cr

Editora técnica: M.Sc. Marcela Quirós G. - marcela.quiros@ucr.ac.cr 
Dr. Juan José Marín Hernández, Catedrático. Director del Centro de Investigaciones Históricas de América Central. Universidad de Costa Rica. Costa Rica. juan. marin@ucr.ac.cr

Dr. David Díaz Arias: Catedrático. Historia Política, Director del posgrado de Historia y Docente de la Escuela de Historia, Universidad de Costa Rica, Costa Rica.david.diaz@ucr.ac.cr

Dr. Ronny Viales Hurtado. Catedrático. Historia Económica y Social. Universidad de Costa Rica. Director de la Escuela de Historia. Costa Rica. ronny. viales@ucr.ac.cr

MSc. Francisco Enríquez. Historia Social. Universidad de Costa Rica. Costa Rica. francisco.enriquez@ucr. ac.cr

Dra. Ana María Botey. Historia de los movimientos sociales. Universidad de Costa Rica. Costa Rica. abotey@gmail.com

\section{Miembros del Consejo Asesor Internacional:}

Dr. José Cal Montoya. Universidad de San Carlos de Guatemala. Guatemala. jecalm@correo.url.edu.gt

Dr. Juan Manuel Palacio. Universidad Nacional de San Martín. Argentina.jpalacio@unsam.edu.ar

Dr. Eduardo Rey. Universidad de Santiago de Compostela. España. ereyt@usc.es

Dr. Heriberto Cairo Carou. Departamento de Ciencia Política y de la Administración III - Universidad Complutense de Madrid. España. hcairoca@cps.ucm.es

Dra. Rosa de la Fuente. Departamento de Ciencia Política y de la Administración III Universidad Complutense de Madrid. España. rdelafuente@cps. ucm.es

Dr. Javier Franzé. Departamento de Ciencia Política y de la Administración III Universidad Complutense de Madrid. España. javier.franze@cps.ucm.es

Dr. Jaime Preciado Coronado Departamento de Estudios Ibéricos y Latinoamericanos. Universidad de Guadalajara. México.japreco@hotmail.com

Dr. Gerónimo de Sierra. Vicerrector de la Universidade Federal da Integração Latino-Americana (UNILA) y Departamento de Sociología, Facultad de Ciencias

Sociales de la Universidad de la República. Uruguay. geronimo@fcs.edu.uy

Dr. Antonio Palazuelos. Departamento de Ciencia Política y de la Administración III - Universidad Complutense de Madrid. España. palazuelosa@cps. ucm.es

Dr. Werner Mackenbach. Universidad Potsdam. Alemania.werner.mackenbach@uni-potsdam.de

Dr. Guillermo Castro. Ciudad del Saber Panamá. Panamá.gcastro@cdspanama.org

Dra. Natalia Milanesio. University of Houston. Estados Unidos.nmilane2@Central.UH.EDU

Dr. Ricardo González Leandri. Consejo Superior de Investigaciones Científicas - España. España. rgleandri@gmail.com

Dra. Mayra Espina. Centro de Estudios Psicológicos y Sociológicos, La Habana. Cuba.mjdcips@ceniai.inf.cu

Dra. Montserrat Llonch. Departamento de Economía e Historia Económica Universidad Autónoma de Barcelona. España. montserrat.llonch@uab.es

Dra. Estela Grassi. Universidad de Buenos Aires. Argentina. estelagrassi@gmail.com

Dra. Yolanda Blasco. Universidad de Barcelona. España. yolandablasco@ub.edu

Dr. Alfredo Falero. Departamento de Sociología. Universidad de la República. Uruguay. alfredof@adinet. com.uy

Portada:

Fotografía: ( Collage "Campesinos en la producción de dulce en un trapiche de Rincón de Mora, Ran Ramón, 1987" ; "Panorámica del Antiguo Palacio Municipal, Antigua Iglesia, Parque y Kiosco de San Ramón, hacia 1920; y Escuela Jorge Washinton, San Ramón hacia 1955). Estas fotografías pertenecen a la Colección Fotográfica del Museo Regional de San Ramón- UCR. Montaje fotográfico: Lic. Juan Gabriel Madrigal Cubero ).

\section{Equipo Técnico Editorial:}

Diagramación y

Edición técnica:

M.Sc. Marcela Quirós Garita. marcela.quiros@ucr.ac.cr

Soporte técnico: Kevin Trejos Vargas

Revisión filológica: Baruc Chavarría Castro 
"Diálogos Revista Electrónica de Historia" se publica desde octubre de 1999.

\section{Diálogos está en los siguientes repositorios:}

Dialnet

http://dialnet.unirioja.es/servlet/

revista?tipo_busqueda=CODIGO\&clave_revista $=3325$

\section{Latindex}

http://www.latindex.unam.mx/larga.php?opcion=1\&folio=12995;

\section{UCRindex}

http://www.revistas.ucr.ac.cr

\section{Scielo}

http://www.scielo.cll

\section{eRevistas}

http://www.erevistas.csic.es/

\section{REDALYC}

http://redalyc.uaemex.mx/src/inicio/FrmBusRevs2.jsp?iEdoRev=2\&cvepai=11;

\section{LANIC}

http://lanic.utexas.edu/la/ca/cr/indexesp.html;

Repositorio de Revistas Universidad de Costa Rica

http://www.latindex.ucr.ac.cr/

Directorio y recolector de recursos digitales del

Ministerio de Cultura de España

http://roai.mcu.es/es/inicio/inicio.cmo

DOAJ Directory of open access \& Hybrid journals

http://www.doaj.org/doaj?func=byTitle\&hybrid=1\&query=D

Biblioteca de Georgetown

http://library.georgetown.edu/newjour/d/msg02735.htm

Asociación para el Fomento de los Estudios Históricos en Centroamérica

http://afehc.apinc.org/index.php?action=fi_aff\&id=1774

Universidad de Saskatchewan, Canadá

https://library.usask.ca/ejournals/view/1000000000397982

Monografias

http://www.monografias.com/Links/Historia/more12.shtml

\section{Hispanianova}

http://hispanianova.rediris.es/general/enlaces/hn0708.htm

Universidad del Norte, Colombia

http://www.uninorte.edu.co/publicaciones/memorias/enlaces.htm

Universidad Autónoma de Barcelona

http://seneca.uab.es/historia/hn0708.htm

Repositorio Invenia - Gestión del Conocimiento http://www.invenia.es/oai:dialnet.unirioja.es:ART0000086144

\section{Enlace Académico}

http://www.enlaceacademico.org/biblioteca/

revistas-en-formato-digital-centroamerica/

\section{Electronic Resources}

http://sunzi1.lib.hku.hk/ER/detail/hkul/3987318

Revistas académicas en texto completo http://web.prw.net/ vtorres/

Diálogos se anuncia en las siguientes instituciones y sitios académicos:

Maestroteca

http://www.maestroteca.com/detail/553/dialogos-revista-electronica-de-historia.html

Biblioteca de Georgetown

http://library.georgetown.edu/newjour/d/msg02735.htm

Asociación para el Fomento de los Estudios Históricos en Centroamérica

http://afehc.apinc.org/index.php?action=fi_aff\&id=1774

Universidad de Saskatchewan, Canadá

https://library.usask.ca/ejournals/view/1000000000397982

Monografias

http://www.monografias.com/Links/Historia/more12.shtm

Hispanianova

http://hispanianova.rediris.es/general/enlaces/hn0708.htm

Universidad del Norte, Colombia

http://www.uninorte.edu.co/publicaciones/memorias/enlaces.html

Universidad Autónoma de Barcelona

http://seneca.uab.es/historia/hn0708.htm

Repositorio Invenia - Gestión del Conocimiento

http://www.invenia.es/oai:dialnet.unirioja.es:ART0000086144

Enlace Académico

http://www.enlaceacademico.org/biblioteca/

revistas-en-formato-digital-centroamerical

\section{Electronic Resources}

http://sunzi1.lib.hku.hk/ER/detail/hkul/3987318

Revistas académicas en texto completo http://web.prw.net/ vtorres/

Diálogos Revista de Historia está catalogada por Sherpa Romeo como una revista verde.

La revista electrónica Diálogos es financiada por Vicerrectoría de Investigación de la Universidad de Costa Rica

Citado en: Scielo Dialnet - eRevistas - UCRindex Latindex - REDALYC - DOAJDirectorio y recolector de recursos digitales del Ministerio de Cultura de España

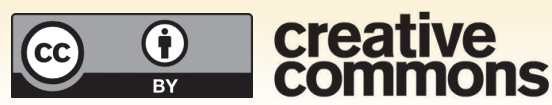




\title{
CONDICIÓN ECONÓMICA E INSTITUCIONAL DE LAS POBLACIONES ADYACENTES A LA RESERVA BIOLÓGICA ALBERTO MANUEL BRENES
}

\author{
ECONOMIC AND INSTITUTIONAL CONDITIONS ON TOWNS \\ ADJACENTS TO ALBERTO MANUEL BRENES BIOLOGICAL \\ RESERVE
}

\begin{abstract}
Ismael G. Guido Granados

\section{Palabras clave}

Área de amortiguamiento, área protegida, conservación biológica, indicadores económicos, indicadores institucionales.
\end{abstract}

\section{Keywords}

Buffer zone, protected area, biodiversity conservation, economic indicators, institutional indicators

Fecha de recepción: 7 de febrero, 2014 - Fecha de aceptación: 4 de agosto, 2014

\begin{abstract}
Resumen
El conocimiento que se tenga sobre las condiciones económicas e institucionales que presentan las comunidades vecinas a las áreas protegidas es fundamental para consolidar el esfuerzo de conservación que se esté ejecutando en ellas y también para alcanzar las mejores condiciones de los habitantes de las comunidades. De este modo, se presenta la realidad que sobre estas temáticas muestra la periferia de la Reserva Biológica Alberto Manuel Brenes, en donde el ingreso económico per cápita es tan bajo que lleva a que la población se ubique en condición de pobreza extrema. A la vez no existe en estos lugares un adecuado manejo y disposición final de los residuos sólidos. En el ámbito institucional existe una amplia cobertura del servicio eléctrico, pero no lo es así para el acceso a telefonía tanto fija como móvil y a Internet.
\end{abstract}

\begin{abstract}
The knowledge about the economic and institutional conditions in the communities around the protected areas is essential to strength the conservation effort that is running into them and also to achieve the best conditions of the inhabitants of the communities. Thus, the reality shows on these topics presents the periphery of the Alberto Manuel Brenes Biological Reserve, where per capita income is so low that leads the population to locate in extreme poverty. Meanwhile in these places there is no proper management and disposal of solid waste. At institutional level there is a wide electricity coverage, but not so for access to telephone and mobile telephone, and Internet.
\end{abstract}




\section{CONDICIÓN ECONÓMICA E INSTITUCIONAL DE LAS POBLACIONES ADYACENTES A LA RESERVA BIOLÓGICA ALBERTO MANUEL BRENES}

\section{INTRODUCCION}

Desde la década de los setenta, Costa Rica apostó por la conservación de los recursos naturales. Este proceso inicia con la creación del Sistema de Parques Nacionales, estableciéndose en setiembre de 1970 los primeros parques nacionales estatales: volcán Poás, Tortuguero y Cahuita, y Santa Rosa en marzo de 1971 (Boza, 1988).

Con el pasar de los años, estos espacios fueron ganando más relevancia en la dinámica de la economía nacional, al presentarse en la década de los noventa un importante aumento en la visitación del turismo naturalista a las áreas protegidas, pero a pesar de estos cambios, no se han realizado los esfuerzos para reinvertir parte de las ganancias generadas en las áreas silvestres protegidas estatales (Adamson, 2008). La falta de reinversión se refleja en la carencia de pago de tierras que se presenta en la mayoría de las áreas silvestres establecidas, ya que un 15\% de los parques nacionales y un $46 \%$ de las reservas biológicas todavía están en manos privadas, a pesar de ser las principales y más restringidas categorías de manejo (Proyecto Estado de la Nación, 2003).

Para combatir los inconvenientes que provocan este tipo de vacíos presentes en la conservación, se procura que en los terrenos ubicados en la periferia de las áreas protegidas, se establezcan zonas de amortiguamiento. Estas deben abarcar los aspectos críticos para la conservación, considerando los beneficios directos del área protegida a las comunidades más cercanas (agua para consumo humano y agrícola) y las amenazas más inmediatas a los recursos que se protegen (crecimiento de la frontera agrícola, actividades ilegales, incendios forestales, contaminación del suelo, del agua y del aire por agroquímicos) (Morales y Bermúdez, 2002).

La finalidad de estos espacios es sumar protección al área protegida y tomando en cuenta que es común que en ellos se asienten comunidades, en algunas circunstancias también buscan compensar a los lugareños por la pérdida de acceso a los recursos que esta posee. Por ejemplo, actualmente se reconoce que algunas áreas protegidas representan una limitación para que las comunidades locales puedan continuar con sus formas tradicionales de aprovechamiento de los recursos naturales, así como también hay especies que se protegen y que causan impactos negativos en las actividades productivas de las poblaciones locales, por lo que es esencial desarrollar una relación de mutuo beneficio con los pobladores vecinos al área silvestre protegida (García, 2002). 
En el caso de la Reserva Biológica Alberto Manuel Brenes (ReBAMB), los primeros asentamientos humanos que se establecieron en su periferia datan de finales del siglo XIX y comienzos del XX. En el año 1906 se funda Cedral de Miramar, ya en 1924 se tiene conocimiento de los poblados San Lorenzo y Colonia Palmareña. Posteriormente, en 1941, ya se reportan los poblados de Las Rocas, San Jorge y San Roque, estos ubicados en el extremo noroeste de la cuenca del río San Lorenzo (Vargas, 1978).

Partiendo de estas referencias se tiene como objetivo mostrar la condición económica e institucional que presentan las poblaciones adyacentes a la ReBAMB y cómo influyen en el proceso de conservación que se da en esta área protegida.

\section{MATERIALES Y MÉTODOS}

\section{Área de estudio:}

El área protegida conocida como Reserva Biológica Alberto Manuel Brenes (ReBAMB) fue oficialmente creada el 10 de junio de 1975 bajo la categoría de manejo y nombre de Reserva Forestal de San Ramón. Esta categoría permite que se puedan dar planes de aprovechamiento forestal y se aplica en espacios cubiertos de bosque en la mayor parte de su extensión, que contenga amplias zonas apropiadas para la producción de madera, agua, forraje, vida silvestre o recreación al aire libre. Puede contener algunos rasgos excepcionales, pero no muy extensos ni muy importantes a escala nacional (Sánchez, 2000).

Posteriormente, en 1991 se promulgó el Decreto Ejecutivo que cambió su categoría de manejo a Zona Protectora, la cual es un área constituida por bosques y terrenos de aptitud forestal, cuyo objetivo principal es la protección del suelo, la regulación del régimen hidrológico, mantener o mejorar la calidad y cantidad de los recursos hidrográficos, la conservación de las cuencas hidrográficas y del ambiente. En sí, bajo esta categoría se tienen áreas que no encierran ningún rasgo único o sobresaliente de carácter natural o ecológico, son de escaso valor científico y potencial recreativo, pueden contener productos madereros aprovechables, que no podrían utilizarse sin perjudicar la calidad o cantidad del agua; además, puede contener asentamientos humanos y algunas zonas alteradas. Por las carencias que presentan las categorías mencionadas, es que en el año de 1993, a través de la Sede de Occidente de la Universidad de Costa Rica y de la Asociación Ramonense para la Conservación del Ambiente (ARCA), se promueve el cambio definitivo en la categoría de manejo a Reserva Biológica (Sánchez, 2000).

La ReBAMB se ubica en el extremo sur de la Cordillera de Tilarán, en la cuenca alta del río San Lorenzo, en el distrito Los Ángeles de San Ramón, 
provincia de Alajuela. Cuenta con una superficie de 7.800 hectáreas y un perímetro de $35 \mathrm{~km}$ de longitud, alrededor de los cuales se asientan, entre otras, las comunidades expuestas en este documento: Corazón de Jesús en Puntarenas, Cedral y Zapotal en Montes de Oro, junto con Los Criques, Parcelas y Colonia Palmareña en San Ramón de Alajuela (Guido, 2007). Para efectos de la investigación se tomó como criterio de escogencia la cercanía de estas comunidades al área protegida, pues se ubican en distintos puntos de su periferia, además de que forman parte de los frentes de colonización que históricamente han ejercido presión sobre la

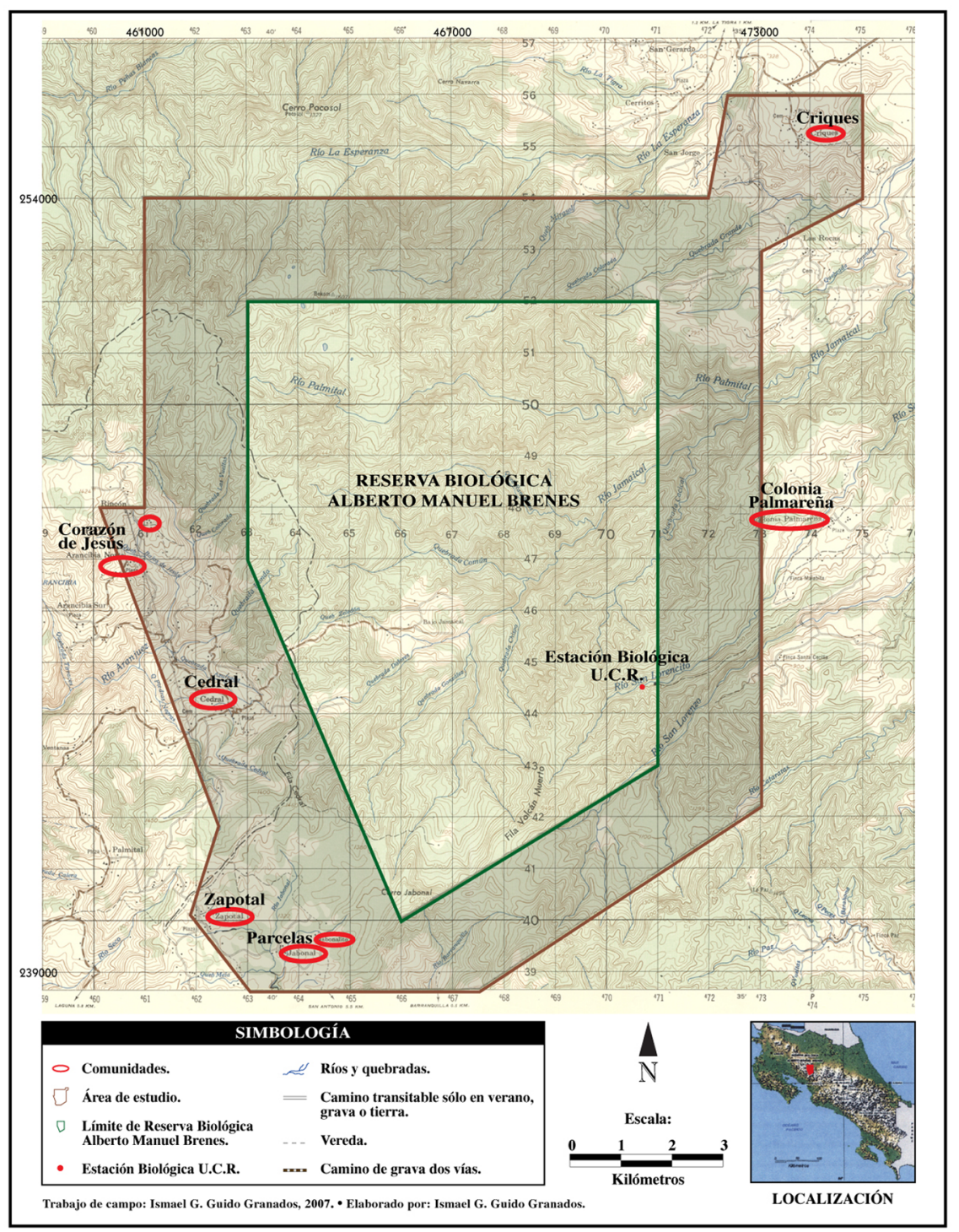

Figura 1. Área de estudio y comunidades ubicadas en la periferia de la Reserva Biológica Alberto Manuel Brenes. Fuente: Modificado de (Instituto Geográfico Nacional, 1994, Hoja San Lorenzo, escala 1:50000). 
ReBAMB (Artavia, 2005). Se entiende como periferia los dos primeros kilómetros alrededor de los límites oficiales de esta Reserva, con excepción de los sectores de Los Criques y de Zapotal, en que el rango se amplía para tomarlos en cuenta (Figura 1).Cedral está ubicado 28 kilómetros al noreste de la ciudad de Miramar de Montes de Oro, posee una población cercana a los 350 habitantes. Zapotal de Miramar, en el costado suroeste de la ReBAMB, cuenta con 140 personas pobladoras y Corazón de Jesús, al noroeste, con aproximadamente 200 habitantes. Todas estas comunidades presentan actividades económicas principalmente de tipo agrícola y ganadera, aunque recientemente se han construidos proyectos turísticos de pequeña escala en estos sitios (Guido, 2007).

Por su parte, Los Criques se ubica al norte del distrito de Los Ángeles, cantón de San Ramón, cuenta con una población de 300 habitantes. En las comunidades de Parcelas y Colonia Palmareña en San Ramón, se ha presentado un proceso de despoblamiento que ha llevado a que tanto el número de habitantes como de servicios que se brinda en ellas hayan disminuido considerablemente. En Parcelas, ubicada en el costado suroeste de la ReBAMB, habitan 33 personas; mientras en Colonia Palmareña, ubicada en el costado este, tan solo viven 11.

\section{METODOLOGÍA}

Durante la etapa de investigación fueron analizados 22 indicadores propuestos por el Observatorio del Desarrollo (ODD) de la Universidad de Costa Rica (2002). Debido a que estos indicadores están diseñados para un análisis a escala nacional, se tomaron aquellos que se pudiesen ajustar a una escala más baja, como en este caso la región tipo rural que conforma la periferia de la ReBAMB.

Los indicadores seleccionados fueron siete del parámetro ambiental, relacionados al uso forestal, agrícola o urbanístico del suelo, a la presencia de áreas protegidas en la zona, a la tala autorizada de árboles y a la presencia de especies vegetales y animales en peligro de extinción, para esto se empleó el análisis de imágenes aéreas, visitas de campo y revisión de expedientes en el Ministerio de Ambiente y Energía. En el ámbito social se analizaron ocho indicadores, relacionados a nivel de pobreza y desempleo, uso de tanques sépticos, población con acceso a agua potable, cobertura de la seguridad social, nivel de alfabetización y cobertura de la escolarización en primaria y secundaria. Para esto se empleó entrevistas a actores claves, revisión de archivos de los Equipos Básicos de Atención Integral en Salud (EBAIS) de las comunidades, así como la aplicación de encuestas (Guido, 2007).

Además, se midieron los indicadores que se presentan en este documento, a saber, tres del área económica, respecto a la separación de residuos para enviar 
a reciclar, otro sobre la reutilización de residuos y el promedio de ingreso económico mensual per cápita por familia. Finalmente se incluyen cuatro del componente institucional, relacionados con el acceso a Internet, servicio de electricidad, así como a la telefonía fija y celular. Para la obtención de los datos de estos últimos, entre setiembre de 2006 y agosto de 2007, se dio la aplicación de 191 entrevistas en igual número de familias, con lo que se obtuvo información de un $72 \%$ de los 1.034 habitantes residentes en la zona de estudio (Guido, 2007).

Para realizar el análisis de los datos se utilizó el Índice Aproximado de Sostenibilidad (IAS), elaborado por Gutiérrez-Espeleta (1994), específicamente aplicando las herramientas para calificar cada parámetro que lo conforma, en este caso el subíndice económico (IAS $-\mathrm{C}_{\mathrm{ECt}}$ ) y el institucional (IAS - $\mathrm{C}_{\mathrm{INSt}}$ ).

Iniciando con el cálculo de la calificación de cada parámetro, de la siguiente manera:

$\mathrm{C}_{\mathrm{kt}}=$ Calificación del parámetro $\mathrm{k}$ en el año t, definido como:

$\mathrm{I}_{\mathrm{kt}}=$ Número de indicadores que estiman el parámetro $\mathrm{k}$ en el año $\mathrm{t}$

$\mathrm{V}_{\mathrm{ikt}}=$ Valor $(-2,2)$ del i-ésimo indicador que estima a k para el año $\mathrm{t}$

$\mathrm{C}_{\mathrm{kt}}=\frac{1}{4 \times \mathrm{I}_{\mathrm{kt}}}\left\{\sum^{\left.1_{\mathrm{kt}}{ }_{\mathrm{i}=1} \mathrm{~V}_{\mathrm{ikt}}\right\}}+1 / 2\right.$

Finalmente, el IAS se estima mediante la siguiente fórmula:

$$
\mathrm{IAS}_{\mathrm{t}}=\frac{\sum_{\mathrm{k}=1}^{4} \mathrm{C}_{\mathrm{kt}} \mathrm{I}_{\mathrm{kt}}}{\sum_{\mathrm{k}=1}^{4} \mathrm{I}_{\mathrm{kt}}}
$$

Para efectos de interpretación, el resultado del IAS y de cada uno de sus componentes debe variar entre 0 y 1 . Al arrojar valores cercanos o iguales a 1 se encuentra en una condición idónea y por el contrario al ser iguales o cercanos a 0 se encuentran en una condición totalmente inadecuada.

\section{RESULTADOS}

Se evaluaron tres indicadores económicos en la periferia de la ReBAMB, lo cual arrojó, según el IAS - $\mathrm{C}_{\mathrm{ECO}}$, un valor de 0 . Por su parte, fueron cuatro los 


\section{Tabla 1}

MANEJO DE RESIDUOS SÓLIDOS EN LA PERIFERIA DE LA RESERVA BIOLÓGICA

ALBERTO MANUEL BRENES, 2007

\begin{tabular}{lcc}
\hline FORMA DE DISPOSICIÓN DE RESIDUOS & FAMILIAS ANALIZADAS & PORCENTAJE OBTENIDO \\
\hline Sólo incineran & 27 & 14,1 \\
Sólo entierran & 51 & 26,7 \\
Entierran e incineran & 57 & 29,8 \\
Incineran y elaboran abono orgánico & 21 & 11 \\
Entierran y elaboran abono orgánico & 11 & 5,8 \\
Servicio privado de recolección & 6 & 3,1 \\
No respondieron & 18 & 9,5 \\
Total & 191 & 100 \\
\hline
\end{tabular}

\section{Tabla 2}

CANTIDAD DE TELÉFONOS EN LAS COMUNIDADES DE LA PERIFERIA DE LA RESERVA BIOLÓGICA ALBERTO MANUEL BRENES, 2007.

\begin{tabular}{lcccc}
\hline COMUNIDAD & DOMICILIARIOS & PÚBLICOS & CELULARES (MÓVILES) & $\begin{array}{c}\text { POBLACIÓN } \\
\text { ANALIZADA }\end{array}$ \\
\hline Los Criques & 21 & 4 & 10 & 160 \\
Colonia Palmareña & 0 & 0 & 2 & 6 \\
Parcelas & 0 & 0 & 7 & 30 \\
Zapotal & 0 & 4 & 7 & 82 \\
Cedral & 63 & 4 & 10 & 326 \\
Corazón de Jesús & 22 & 2 & 4 & 139 \\
Totales & 105 & 14 & 40 & 743 \\
\hline
\end{tabular}

indicadores evaluados en relación al ámbito institucional, lo que arrojó un valor de 0,312 , según el IAS $-\mathrm{C}_{\mathrm{INSt}}$.

En relación al parámetro económico, dos de los indicadores medidos en este análisis están relacionados con el manejo de los residuos sólidos, estos fueron la separación de residuos para reciclaje y el otro la reutilización de residuos, los resultados se presentan en la Tabla 1.

En relación al promedio de ingreso económico mensual per cápita por familia, se obtuvo como resultado que este ingreso es de $\phi 14.625$, lo que es una condición totalmente inaceptable para este indicador.

En relación al parámetro institucional, se midió el indicador llamado líneas 
telefónicas fijas por cada cien habitantes y el indicador líneas telefónicas celulares (móviles) por cada cien habitantes. En las Tablas 2 y 3 se desglosa la cantidad y densidad de teléfonos públicos, domiciliarios y celulares presentes en la periferia de la ReBAMB: los valores obtenidos, 14 líneas fijas y 5,3 líneas celulares por cada 100 habitantes, están por debajo de los valores establecidos para Costa Rica.

Dentro de los indicadores institucionales, el que presenta el mayor rezago es el referente al acceso a Internet. Además, en ninguna de las comunidades se brinda este servicio, ya sea en forma de los denominados café Internet o algún otro tipo de negocio, los datos obtenidos se presentan en la Tabla 4.

\section{Tabla 3}

DENSIDAD TELEFÓNICA POR CADA CIEN HABITANTES EN LA PERIFERIA

DE LA RESERVA BIOLÓGICA ALBERTO MANUEL BRENES, 2007.

\begin{tabular}{lcc}
\hline TIPO DE SERVICIO & CANTIDAD DE LINEAS & LÍNEAS / 100 HABITANTES \\
\hline Fijo (domiciliario) & 105 & 14 \\
Celular (móvil) & 40 & 5,3 \\
\hline
\end{tabular}

\section{Tabla 4}

FAMILIAS RESIDENTES EN LA PERIFERIA DE LA RESERVA BIOLÓGICA ALBERTO MANUEL BRENES, 2007.

\begin{tabular}{lccc}
\hline \multicolumn{1}{c}{ COMUNIDAD } & FAMILIAS CON ACCESO & PORCENTAJE & FAMILIAS \\
& A INTERNET & OBTENIDO & ANALIZADAS \\
\hline Los Criques & 1 & 0,5 & 38 \\
Colonia Palmareña & 0 & 0 & 4 \\
Parcela & 0 & 0 & 8 \\
Zapotal & 0 & 0 & 23 \\
Cedral & 3 & 1,6 & 89 \\
Corazón de Jesús & 0 & 0 & 29 \\
Total & 4 & 2,1 & 191 \\
\hline
\end{tabular}


Tabla 5

COBERTURA DE SERVICIO ELÉCTRICO EN LAS COMUNIDADES DE LA PERIFERIA DE LA RESERVA BIOLÓGICA ALBERTO MANUEL BRENES, 2007.

\begin{tabular}{lccc}
\hline \multicolumn{1}{c}{ COMUNIDAD } & POBLACION ABASTECIDA & $\begin{array}{c}\text { COBERTURA } \\
\text { POBLACIONAL }\end{array}$ & POBLACION ANALIZADA \\
\hline Los Criques & 160 & $100 \%$ & 160 \\
Colonia Palmareña & 0 & $0 \%$ & 6 \\
Parcelas & 30 & $100 \%$ & 30 \\
Zapotal & 82 & $100 \%$ & 82 \\
Cedral & 326 & $100 \%$ & 326 \\
Corazón de Jesús & 139 & $100 \%$ & 139 \\
Total & 737 & $99,2 \%$ & 743 \\
\hline
\end{tabular}

La Tabla 5, muestra la cobertura de servicio eléctrico que se presenta en las comunidades de la periferia de la ReBAMB.

\section{DISCUSIÓN}

El bienestar económico de todos los miembros de la sociedad debe ser una prioridad, mas no la única, en el proceso de instituir actividades productivas que sean beneficiosas a la economía, equitativas para la sociedad y ambientalmente responsables. Esto como una manera de permitir el pleno desarrollo individual de las personas y garantizar el éxito de la estructura social, es por ello que todas las actividades productivas deben estar involucradas en el manejo adecuado de los recursos naturales y en el manejo responsable de los residuos ocasionados en sus procesos productivos.

En el caso de los primeros indicadores económicos, la separación de materiales para reciclaje y la reutilización de residuos, se obtuvieron valores preocupantemente bajos, ya que con base en estimaciones realizadas para el país, el promedio de producción de basura es de 1,2 kg diario por habitante (PRESOL, 2007), y dado que en la zona viven poco más de 1.000 habitantes, de mantenerse esta estimación, se produciría más de una tonelada diaria de residuos sólidos, los cuales son manejados de forma inadecuada (Tabla 1). El manejo inadecuado de 
residuos inicia con el hecho de que en ninguna de las comunidades analizadas existe el servicio recolector de basura por parte de la municipalidad respectiva. Dadas las deficiencias en este aspecto, las familias acostumbran enterrar o incinerar en sus propiedades los residuos que producen (Tabla 1).

Otro inconveniente en el manejo de residuos se presenta debido a que no existen en la zona, al momento del estudio, iniciativas de instituciones públicas o privadas para fomentar la separación, reutilización y reciclaje de materiales sólidos de desecho.

Para el indicador separación de material para reciclaje, el valor que arroja es bajo en cuanto a cantidad, debido a que en un $90 \%$ de los hogares visitados no se realizan prácticas de separación de materiales, y en los que se hace es solamente para una baja cantidad y predominan las prácticas para manejo de residuos descritas en la Tabla 1. Además, en ambos casos, separación para reciclaje o reutilización, la gran mayoría de residuos no están recibiendo un tratamiento adecuado, ya que no existen centros de acopio ni rellenos sanitarios en la zona analizada.

Los valores obtenidos para ambos indicadores reflejan la situación que se presenta en el país, en donde la actividad del reciclaje es propia de un sector no formal y poco estructurado. Esta es desarrollada por empresarios de diversa magnitud, quienes generalmente son intermediarios entre el proveedor y el reciclador. Por lo tanto, se carece de un sistema nacional estructurado para la recuperación, que estimule una cultura de reducción, reuso y reciclaje de los residuos (Observatorio del Desarrollo, 2002). Por lo que se puede afirmar que en la zona el reciclaje y reutilización de residuos separados y recuperados es mínimo con relación a su potencial.

Se debe entender que la mayor parte de la responsabilidad en los procesos de reciclaje recae precisamente en el consumidor, quien es el que debe informarse y decidir acerca del tipo de producto que desea comprar y del tipo de residuo que va a producir su consumo. Los residuos sólidos descartados generan costos ambientales que deberían reflejarse en el precio que paga el consumidor, quien debe tener la conciencia de pagar un poco más por un producto que posea un empaque reciclable. Se debe tener presente que en ningún proceso de reciclaje se logra recuperar la totalidad del material utilizado (Field y Field, 2003).

Aunque si bien esta no es aún la realidad que se está presentando en la periferia de la ReBAMB, se debe implementar un plan estratégico para el manejo de los residuos sólidos, el cual incluya la separación de materiales para ser reciclados y reutilizados. Ante todo porque tal y como lo manifiestan muchos habitantes, además de ser evidente en el tipo de negocios que inician en la zona, el futuro de estas comunidades se encuentra en el desarrollo de la industria turística, lo cual acarrearía un aumento en la población residente junto con la población que tan solo 
estaría de paso por periodos variados, y por ende esto conlleva a que se aumente el total y variedad de residuos sólidos producidos, por lo que se deben tomar medidas previsoras para una futura problemática de esta índole.

Por otra parte, es de vital importancia conocer la situación económica de las familias que residen en el espacio de interés, esto debido a que son estas personas las que deben lidiar con la realidad cotidiana y son precisamente las que están interactuando y haciendo uso de los recursos naturales ahí presentes.

Por estas razones se midió el indicador denominado promedio de ingreso económico mensual per cápita por familia. Para las familias presentes en la periferia de la ReBAMB, el resultado demuestra que el valor de ingreso es sumamente bajo ya que tan solo alcanza el monto promedio de $\phi 14.625$ por persona por familia por mes, lo que a la vez colocaría a toda la población analizada en una condición de pobreza extrema, lo cual sería una forma de interpretar la mala distribución de los recursos económicos que impera en la actualidad. La razón radica en que al ser estas comunidades de tipo rural las actividades a las que se dedican sus habitantes son principalmente las agropecuarias. Debe destacarse el hecho de que la pobreza en esta zona se manifiesta como la escasez de dinero circulante y no como la presencia de tugurios o indigencia, que son expresiones comunes en otras zonas del país (Guido, 2007).

Las carencias económicas presentes en la zona y las limitaciones asociadas se han reportado en distintos momentos; por ejemplo, Sandoval (1995) indica que para los habitantes de sitios como Bajo La Paz y Zapotal de Miramar, la ReBAMB y sus alrededores representan importancia como suministradores de leña, caza y pesca. Por su parte, Fernández y Saborío (2002) señalan que para algunas familias de la zona los recursos animales extraídos de la Reserva constituyen la única fuente de proteína que consumen.

Este tipo de situaciones conllevan un choque directo de intereses entre las comunidades y la categoría de manejo del área protegida, ya que de acuerdo con Thelen y Dalfet (1979) dentro del proceso de conservación de recursos naturales, se define la Reserva Biológica como un área esencialmente inalterada por la actividad humana y que contiene ecosistemas, rasgos o especies de flora y fauna de valor científico, en la cual los procesos ecológicos han podido seguir su curso espontáneo con un mínimo de interferencia, por lo que sus objetivos son exclusivamente la investigación y la conservación, por lo que cualquier otro fin debe considerarse secundario.

Es aquí en donde el manejo de las zonas de amortiguamiento se debe plantear como la búsqueda de formas de relación de beneficio mutuo, en donde la población tenga acceso a mejores condiciones de vida sin detrimento de los esfuerzos de conservación de la biodiversidad (García, 2002). 
La medición de indicadores institucionales, relacionados con el acceso de la población a medios eficaces de información, es de elevada importancia política dado que las telecomunicaciones están estrechamente relacionadas con el desarrollo social, económico e institucional (Observatorio del Desarrollo, 2002). Del análisis de las comunidades asentadas en la periferia de la ReBAMB, se logró obtener que existe en promedio una baja disponibilidad de líneas telefónicas fijas (Tablas 2 y 3 ). El valor obtenido para la telefonía fija domiciliaria dista de los valores nacionales, ya que los datos evidencian un rezago. Por ejemplo, en el año 1997 había en el país 19,5 líneas por cada 100 habitantes (Cordero, 2000), y para el año 2004 este valor era de 31,6 líneas por cada 100 habitantes (Programa de la Sociedad de la Información y el Conocimiento, 2006). Pero a pesar de estas limitaciones cabe resaltar la labor hecha por parte del Instituto Costarricense de Electricidad en este campo, para dotar de este servicio a comunidades que de lo contrario quedarían aisladas del resto del país.

El acceso a líneas telefónicas celulares también es escaso (Tabla 3), en este sentido y en relación con la situación nacional que se presenta en cuanto a la densidad de esta telefonía, se debe tener presente que la cobertura nacional de este servicio en el año 2004 era de 21,7 líneas por cada 100 habitantes (Programa de la Sociedad de la Información y el Conocimiento, 2006). Lo anterior muestra un rezago en esta temática en la zona de estudio, pero se debe tener presente que tal y como se logró comprobar durante el trabajo de campo, en la gran mayoría del área de la periferia analizada la cobertura para telefonía celular de tecnología TDMA o GSM era nula y donde la había era de mala recepción.

$\mathrm{El}$ acceso a Internet es el aspecto que presenta el mayor nivel de rezago, ya que de la totalidad de hogares muestreados, tan solo cuatro de ellos contaban con este servicio. Además en ninguna de las comunidades se brinda este servicio ya sea en forma de los denominados café Internet o algún otro tipo de negocio (Tabla 4). A partir de lo anterior se puede afirmar que solamente un 2,1\% de las familias tiene acceso a esta herramienta tan útil y necesaria en la vida moderna.

Esta situación, aunque es extrema, demuestra la realidad que se presentaba el país en el año 2007, ya que el nivel de acceso a Internet de la población de zonas urbanas era del $85,2 \% \mathrm{y}$, por el contario, en el caso de las comunidades rurales del país este valor era de tan solo un $14,8 \%$. Esto deja de manifiesto el por qué de la poca accesibilidad que se tiene en las comunidades de la periferia de la ReBAMB a este servicio, pero al comparar esta accesibilidad de acuerdo con los valores según las regiones de planificación, se observa que es solo un poco menor a los datos de las dos regiones de interés, que son la Huetar Norte, con un 3,6\%, y la Pacífico Central, con un 3,7\% (Programa de la Sociedad de la Información y el Conocimiento, 2006). 
Este contraste conlleva a que se acentúe la denominada brecha digital, entendiéndose por esta la diferencia en cuanto al acceso y uso de las aplicaciones de las tecnologías de la información y las comunicaciones entre individuos de un mismo país, o entre diferentes países o regiones. De hecho, el concepto de brecha digital está estrechamente relacionado con el nivel de ingreso, género, nivel de educación, lugar de residencia, edad y otras dimensiones socioeconómicas (Monge y Chacón, 2002). Esta afecta a las personas y grupos sociales y suele manifestarse en un desequilibrio entre la productividad y el desarrollo tecnológico, lo cual afecta la competitividad económica de los países y regiones frente a un mundo que, aunque segmentado desde el punto de vista tecnológico, se integra cada vez más en otros ámbitos (Programa de la Sociedad de la Información y el Conocimiento, 2006).

Finalmente, en relación a la cobertura de servicio eléctrico, es el rubro mejor calificado en este análisis, ya que de las comunidades involucradas, solo Colonia Palmareña carece de este servicio (Tabla 5), el nivel de cobertura resulta similar al valor nacional, el cual según Fallas (2001) es cercano a un 98,5\%. Esta alta cobertura permite que los habitantes de esta zona tengan un fácil acceso a la información que se brinda por medio de la radio y televisión. Potencialmente, al existir una alta cobertura de servicio eléctrico y acceso a instituciones de educación, se puede llegar a dar en el mediano plazo una mayor accesibilidad al servicio de Internet. También, se debe tener presente la serie de facilidades que conlleva el poseer este servicio, en aspectos tales como el hogar o en la producción agrícola o industrial.

Dado que en Costa Rica se estableció desde el año 1994 el denominado Sistema Nacional de Áreas de Conservación (SINAC) para manejar los recursos naturales mediante la creación de once áreas de conservación, en las cuales no solo se incluye como su jurisdicción las áreas silvestres protegidas sino la totalidad del país, se debe entender que al ser responsabilidad de este organismo velar por la conservación, es necesario tener datos muy claros sobre la situación, en este caso institucional y económica, que se presenta en cada una de las comunidades que conforman las áreas de conservación, para así saber con certeza las repercusiones que las actividades humanas están teniendo sobre los recursos que se están protegiendo y planificar las medidas correctivas.

En el caso particular de los indicadores institucionales, la importancia que manejan dentro del proceso de conservación radica en la disminución de presiones que se ejerzan en el ambiente por actividades tales como el transporte debido al desplazamiento de personas que podrían realizar trámites por Internet o teléfono. También, se debe rescatar el hecho de que esta serie de servicios que se pueden estar brindando en distintas comunidades, cercanas o no a las áreas protegidas, colaboran en mejorar la calidad de vida de las personas que habitan en estos lugares, lo cual trae entre otros beneficios, que las personas obtengan nuevas opciones productivas, 
con las cuales se puedan dejar de lado las tradicionalmente aplicadas, que traen como consecuencia actividades perjudiciales para el proceso de conservación.

\section{CONCLUSIONES}

En la periferia de la ReBAMB existen serias carencias en lo referente al manejo de los residuos sólidos, ya que hasta la fecha los métodos aplicados son totalmente inadecuados. Lo anterior junto al escaso ingreso económico mensual per cápita conlleva a que la evaluación del parámetro económico se encuentre en una condición precaria.

Se debe incrementar el acceso de la población a medios de comunicación e información eficientes y de bajo impacto en el ambiente, como la telefonía y el Internet.

Finalmente, es posible indicar que los parámetros económico (IAS $-\mathrm{C}_{\mathrm{ECt}}$ ) e institucional (IAS $-\mathrm{C}_{\mathrm{INSt}}$ ) son para los tomadores de decisiones una herramienta de gran utilidad, ya que permiten el monitoreo y planeamiento de las políticas socioeconómicas e institucionales que deben establecerse en la periferia de la ReBAMB.

\section{REFERENCIAS}

Adamson, M. (2008). ¿Son sostenibles las áreas protegidas?: Biorentas y eficiencia del gasto. Revista de Ciencias Económicas, 26(1): 283-306.

Artavia, G. (2005). La evolución de los frentes de colonización agrícola y la tenencia de la tierra en la Reserva Biológica Alberto Manuel Brenes, San Ramón, Alajuela, Costa Rica 1975-2005. (Tesis de Maestría en Geografía). Universidad de Costa Rica, Costa Rica.

Boza, M. (1988). El Sistema de Parques Nacionales de Costa Rica: un ejemplo de posibilidad para la conservación en un país en desarrollo. En E. Guier y G. Martínez. (Eds.). Los recursos naturales y su conservación. (pp. 85-101). Costa Rica: Editorial de la Universidad Estatal a Distancia y Fundación de Parques Nacionales.

Cordero, J. (2000). El crecimiento económico y la inversión: el caso de Costa Rica. Recuperado de http://www.cepal.cl/publicaciones/xml/3/4573/lc11346e.pdf/

Fallas, C. (2001). Distribución eléctrica del Instituto Costarricense de Electricidad (ICE) y su aporte al desarrollo de Costa Rica: 1949-2000. Costa Rica: Colección Patrimonio y Futuro. 
Fernández, A. y Saborío, C. (2002). Estrategias de sobrevivencia de las familias campesinas de la comunidad de Cedral de Miramar Puntarenas: desde una perspectiva del desarrollo sostenible y del trabajo social. (Tesis de Licenciatura en Trabajo Social). Universidad de Costa Rica, Costa Rica.

Field, B. y Field, M. (2003). Economía ambiental. Tercera edición. España: Mc Graw Ill Interamericana.

García, R. (2002). Biología de la conservación: conceptos y prácticas. Costa Rica: Instituto Nacional de Biodiversidad (INBio).

Guido, I. (2007). Estimación del índice aproximado de sostenibilidad en la periferia de la Reserva Biológica Alberto Manuel Brenes, Costa Rica. (Tesis de Maestría en Desarrollo Sostenible). Universidad de Costa Rica, Costa Rica.

Gutiérrez-Espeleta, E. (1994). Indicadores de Sostenibilidad: instrumentos para la evaluación de las políticas nacionales. Revista Ciencias Económicas, 14(2): 37-50.

Monge, R. y Chacón, F. (2002). La brecha digital en Costa Rica: Acceso y Uso de las Tecnologías de la Información y las Comunicaciones. Recuperado de http://www.conicit.go.cr/documentos/ documentos/listadocs/brechadigital.pdf/

Morales, R. y Bermúdez, F. (2002). Plan de Manejo Parque Nacional Volcán Irazú. Costa Rica: Centro Agronómico Tropical de Investigación y Enseñanza (CATIE).

Observatorio del Desarrollo. (2002). Indicadores del Desarrollo Sostenible de Costa Rica, 2002. Costa Rica: Observatorio del Desarrollo, Universidad de Costa Rica y Ministerio del Ambiente y Energía.

PRESOL. (2007). Plan de Residuos Sólidos Costa Rica: Diagnóstico y Áreas Prioritarias. Costa Rica: PRESOL.

Programa de la Sociedad de la Información y el Conocimiento. (2006). Hacia la sociedad de la información y el conocimiento en Costa Rica, Informe 2006. Recuperado de http://www. prosic.ucr.ac.cr/

Proyecto Estado de la Nación. (2003). Estado de la Nación en Desarrollo Humano Sostenible: Noveno informe 2002. Costa Rica: Proyecto Estado de la Nación. 
Sánchez, R. (2000). Reserva Biológica Alberto Manuel Brenes. Costa Rica: Ministerio de Ambiente y Energía.

Sandoval, E. (1995). La reproducción social de las familias ubicadas en el área de amortiguamiento de la Reserva Biológica Alberto Manuel Brenes y su incidencia en la sostenibilidad de los recursos de la zona. (Tesis de Licenciatura en Trabajo Social). Universidad de Costa Rica, Costa Rica.

Thelen, K. y Dalfet, A. (1979). Políticas para el manejo de áreas silvestres. Costa Rica: Editorial de la Universidad Estatal a Distancia.

Vargas, G. (1978). Diagnóstico y recomendaciones para el manejo y ordenamiento de los recursos naturales en la cuenca del río San Lorenzo, Alajuela, Costa Rica. (Tesis de Licenciatura en Geografía). Universidad de Costa Rica, Costa Rica.

\section{SOBRE EL AUTOR}

Ismael G. Guido Granados: Máster en Desarrollo Sostenible - Conservación Biológica. Docente - Investigador de la Universidad de Costa Rica, Sede de Occidente, Sección de Biología. Correo electrónico: ismaelguido@gmail.com 